\title{
Neutrophil NETs: a novel contributor to preeclampsia-associated placental hypoxia?
}

\author{
Anurag Kumar Gupta • Paul Hasler • \\ Wolfgang Holzgreve $\cdot$ Sinuhe Hahn
}

Received: 17 January 2007 / Accepted: 2 April 2007 / Published online: 27 April 2007

(C) Springer-Verlag 2007

\begin{abstract}
Recent studies have suggested that the innate immune system is involved in the pathogenesis of preeclampsia. Its pathogenesis involves neutrophil activation and increased levels of cell-free DNA in the maternal plasma. Activation of neutrophils has recently been shown to induce DNA containing neutrophil extracellular traps (NETs) which trap and kill bacteria. Massive NETs induction by the placentally derived factors (IL-8 and placental micro-debris) and their increased presence in preeclamptic placenta suggest that NETs might be involved in the pathogenesis of preeclampsia. Therefore, increased presence of NETs in preeclampsia may play a role in the deficient placental perfusion associated with this disorder.
\end{abstract}

Keywords Neutrophils $\cdot$ IL-8 $\cdot$ NETs $\cdot$ STBM $\cdot$ Placenta

\section{Introduction}

Although pregnancy seems a happy symbiosis between mother and fetus, in reality, this symbiosis is very complicated. Success of any pregnancy hinges on a mother embracing her fetus immunologically rather than attacking

\footnotetext{
A. K. Gupta $(\bowtie) \cdot$ W. Holzgreve $\cdot$ S. Hahn

Laboratory for Prenatal Medicine,

University Women's Hospital/Department of Research,

University Hospital,

Hebelstrasse 20,

CH-4031 Basel, Switzerland

e-mail: Anurag.Gupta@unibas.ch

P. Hasler

Department of Rheumatology, Kantonsspital Aarau,

Aargau, Switzerland
}

it. Why would a mother's body attack her own fetus? The reason is very obvious; it only belongs partially to the mother. Half of the gene pool of the fetus is contributed by the father; therefore, fetal genes have their own fate. This strangeness can prompt the mother's immune system to counterattack against the fetus. This attack contributes to a whole spectrum of disorders leading to premature birth that can be triggered by a condition called preeclampsia characterized by a rapid elevation in maternal blood pressure. Pathogenesis of this enigmatic disorder seems to involve placenta and overt activation of the maternal innate immune system. In this review, we will focus on the role of maternal innate system, with emphasis on maternal neutrophils, in the pathogenesis of preeclampsia. Moreover, we will discuss how a recently described novel finding, i.e., neutrophils extracellular traps (NETs) formation by activated neutrophils, play an important role in the pathogenesis of preeclampsia.

\section{Pathogenesis of preeclampsia}

Preeclampsia, a severe disorder of late pregnancy, is characterized by elevated blood pressure and proteinuria in previously normotensive pregnant women. It is a leading cause of fetal and maternal mortality worldwide [1, 2]. Preeclampsia is a multisystem disorder of human pregnancy that may be explained by two generalized processes occurring within the maternal and fetal compartments: vasospasm and endothelial dysfunction. It is widely accepted that circulating factors, most likely placental in origin, are responsible for these systemic disturbances. The precise nature of these factors and the mechanism by which a placental disorder may induce these pathophysiologic changes remain unknown. It has been thought to result 
from reduced placental perfusion due to abnormal trophoblast differentiation $[1,2]$. This placental condition is also associated with the elevated release of inflammatory syncytiotrophoblast microvillous membrane microparticles (STBM) and cytokines, which have been proposed to play a role in the overt activation of the maternal innate immune system observed in preeclampsia [3-5].

The STBM are released from the syncytiotrophoblast layer, which is in direct contact with the maternal blood. Therefore, STBM are directly released into the intervillous spaces where they come into the contact with maternal immune cells. It has been suggested that STBM that are released in preeclampsia have altered morphologic characteristics, including areas of syncytial knots attributed to placental hypoxia and areas of aponecrosis associated with loss of STBM [6]. Increased levels of STBM have been reported in the uterine venous and peripheral circulation of women with preeclampsia [7]. It has been shown that STBM have endothelium disrupting activities and can inhibit the proliferation of endothelial cells in culture and inhibit endothelial cell-dependent relaxation of small arteries in vitro [8-10].

Leukocyte activation is increased in preeclampsia compared with normal pregnancies [11, 12]. Therefore, it is possible that after being released into the maternal circulation, STBM come into contact with maternal neutrophils. STBM have been shown to activate neutrophils in vitro [13]. Leukocyte interaction with STBM may amplify endothelial disruption by the release of harmful cytokines and generation of oxygen-free radicals [14]. Reactive oxygen species produced by neutrophils may affect vascular tone directly by contracting smooth muscle tissue and indirectly by inactivating the endothelium-derived relaxing factor or reducing the release of prostacyclin [15].

In addition, we and others have previously shown that preeclampsia is associated with increased transplacental trafficking of fetal cells [16], as well as elevated concentrations of cell-free fetal DNA [17-19] which may occur before onset of symptoms [20-22]. These studies, however, also indicated that cell-free DNA levels of maternal origin were also significantly elevated in preeclampsia and that these levels correlated well with the severity of the disorder [18]. While the results concerning fetal cells and cell-free fetal nucleic acids have been interpreted as further evidence of an underlying placental dysfunction in preeclampsia, those concerning the elevated presence of circulatory DNA of maternal origin have been more difficult to explain, as the source of this material is unclear [23].

\section{Neutrophils extracellular traps}

Recently, Brinkmann et al. [24] described a novel tool with which neutrophils enhance killing of extracellular pathogens while minimizing damage to the host cells. Their data revealed that upon in vitro activation with the pharmacological agent phorbol myristate acetate, IL- 8 or LPS, neutrophils release granule proteins and chromatin to form an extracellular fibril matrix, i.e., NETs, through an active process the molecular mechanism of which remains to be understood in detail [25]. More recently, Brinkmann and colleagues have also shown that not only bacteria but also pathogenic fungi such as Candida albicans induce neutrophils to form NETs that capture and kill C. albicans hyphal as well as yeast-form cells. Therefore, they propose that the NETs could particularly support killing of microbes that are too large to be phagocytosed efficiently. A neutrophil can disarm circumvent this problem by enclosing microbes together with other neutrophils $[25,26]$.

Structurally, NETs are composed of nuclear constituents such as chromatin DNA and histones and granular peptides and enzymes (e.g., elastase and myeloperoxidase) that are normally stored in typical neutrophil granules. DNA is the major structural component of NETs. The treatment of NETs with DNase results in their disintegration, suggesting that DNA, which provides the backbone to the effector proteins, are bound. The NETs produced by the neutrophils consist of smooth fibers with a $15-17 \mathrm{~nm}$ diameter and globular domains of $25-28 \mathrm{~nm}$ diameter [24].

NETs interact with a variety of different pathogens Gram-positive and Gram-negative bacteria as well as fungi and kill them by providing a high local concentration of antimicrobial proteins such as neutrophil elastase, bactericidal permeability-increasing protein (BPI), and histones. NETs have also been shown to play role in the diseases in vivo. Since Brinkmann et al. [24] originally showed the occurrence of NETs in spontaneous human appendicitis and Shigella-induced experimental dysentery in rabbits, NETs have been found to be abundant at sites of infection. Recently, two reports have shown the presence of NETs in the murine models of pneumococcal pneumonia and fasciitis [27, 28]. Moreover, NETs were also found in bovine mastitis where neutrophil phagocytosis and oxidative burst have been shown to be hampered by milk fat and proteins [29].

It has been argued that degrading enzymes of NETs pose a risk for tissue damage [30]. However, after being released into the local environment, which is produced by NETs, these enzymes are less likely to cause damage after secretion. Nevertheless, NETs might have a deleterious effect on the host because of the leakage of nucleic acids, potentially playing a role during the development of autoimmune diseases like lupus erythematosus, a disease associated with autoimmune reactions to the host's own DNA. 

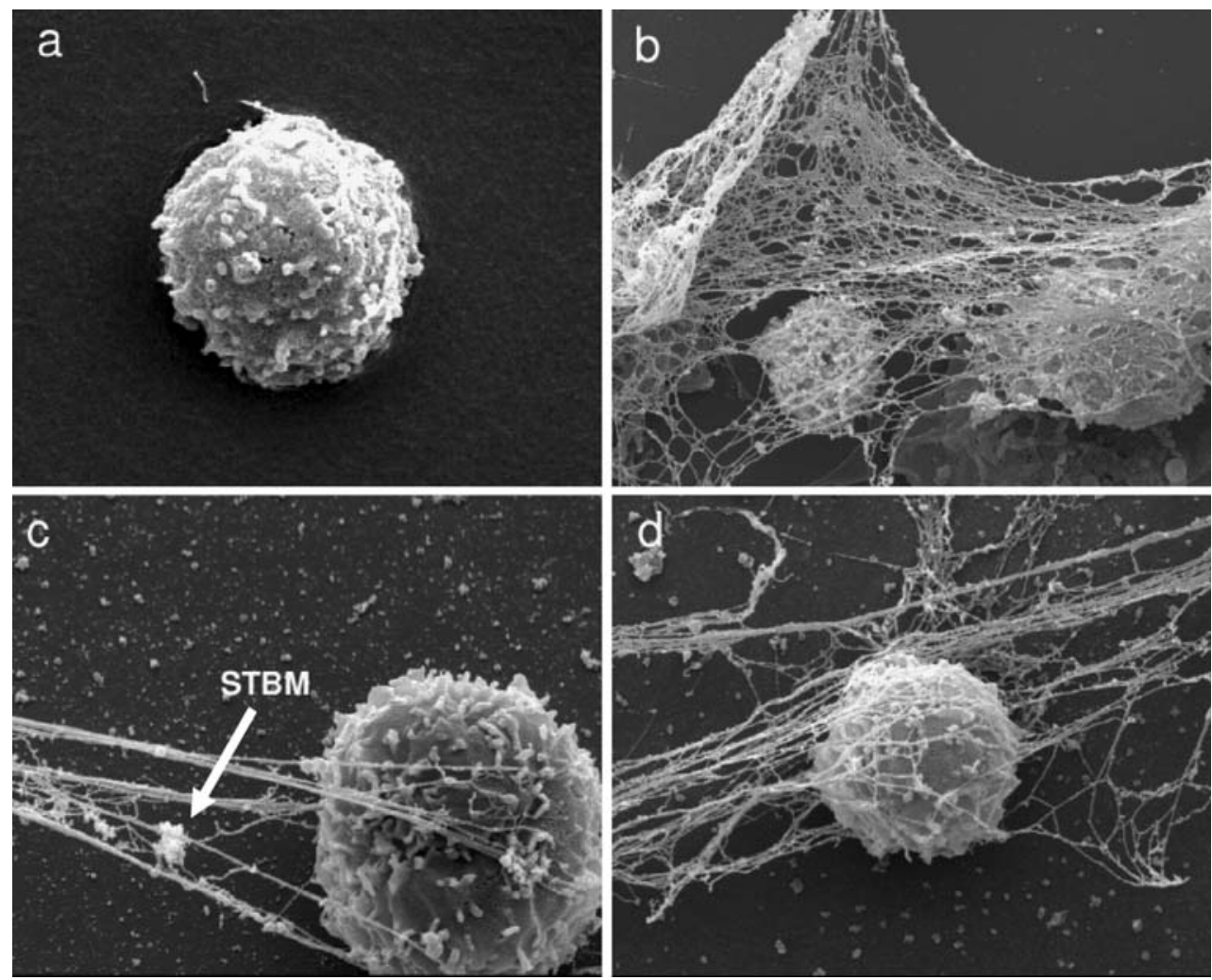

Fig. 1 NETs induction by the activated neutrophils. Untreated neutrophils are round and devoid of NETs (a). Upon activation with PMA (b) STBM (c), and STBM-free villous explants placental conditioned medium (d), neutrophils generated NETs within 30 min of the treatment

\section{Preeclampsia and NETs}

The discovery of the NETs and their nature to release DNA in an extracellular form led us to examine whether placentally derived inflammatory factors can similarly activate neutrophils to generate NETs and whether the presence of such NETs was increased in preeclampsia, as this feature may, hence, link the independent observations concerning the activation of peripheral neutrophils and the elevated presence of maternal circulatory DNA during preeclampsia.

In our in vitro analysis, we examined the capacity of the placentally derived STBM, produced by various methods, and STBM-free soluble factors to activate neutrophils and subsequent NETs generation [31]. Our analysis revealed that placentally derived STBM and soluble factors efficiently activated neutrophils to generate NETs. Similar to the findings of Brinkmann et al., these NETs were rich in DNA, elastase, and histones [31]. Interestingly, we observed that NETs entrapped clusters of the STBM, suggesting that the NETs not only capture large microorganism such as bacteria $(>2 \mu \mathrm{m})$ but also ensnare very small $(<200 \mathrm{~nm})$ inflammatory particles such as STBM (Fig. 1). Among the placentally derived soluble factors, we could confirm that placentally derived IL-8 was responsible for the NETs formation [31]. As STBM, and possibly, placentally-derived IL-8 are released directly by the syncytiotrophoblast into the maternal circulation in the
Fig. 2 Analysis of the placental tissue sections for the presence of NETs. Immunofluorescence staining of the NETs revealed their localization within the villi or adjacent to the syncytiotrophoblast in the normal placenta. The preeclamptic placenta revealed massive presence of NETs that are largely localized within the intervillous space
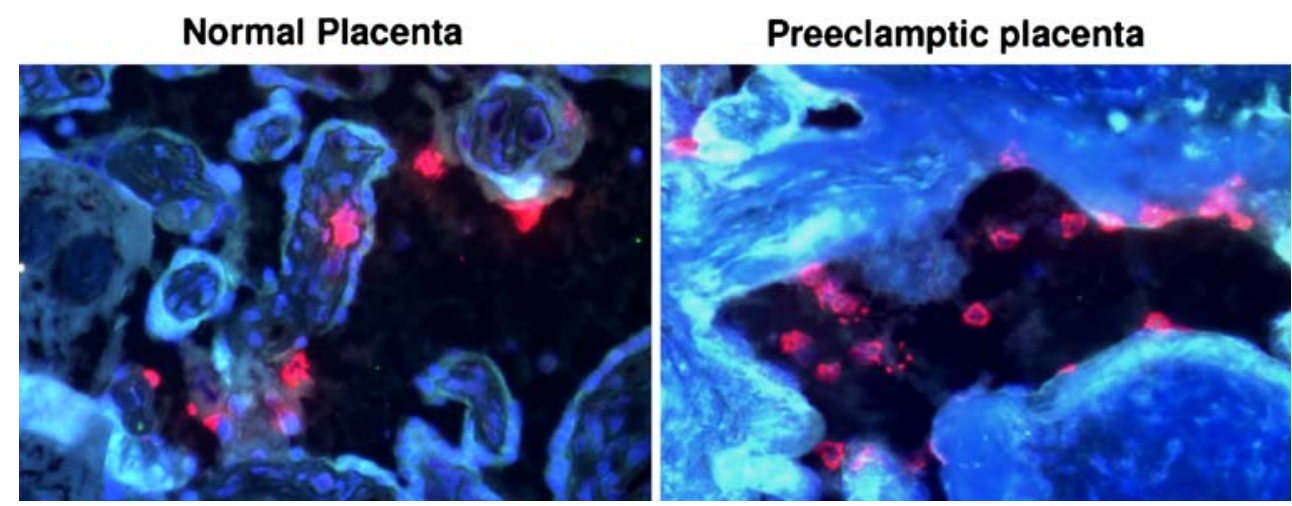
intervillous space, we next examined for the presence of neutrophils NETs in cryosections of placental villous tissue. The presence of NETs in the placentae, as detected by combined staining for DNA, histones, and neutrophilsderived elastase, indicated that their number was quite small in normal placental tissue compared to preeclamptic placentae where vast majority of NETs could be observed. These NETs were also confined to close contact with the syncytiotrophoblast, possibly at the site of immediate release of STBM, as this shedding may involve a budding-off process (Fig. 2) [32].

It is widely accepted that inadequate perfusion of the placenta by maternal blood is a key feature contributing to the development and pathogenesis of preeclampsia. In this regard, the inability of invasive cytotrophoblasts to switch their repertoire of adhesion molecules to resemble that of vascular cells [33] results in inadequate invasion and modification of the maternal spiral arterioles. Consequently, these uterine arteries remain narrow high resistance vessels, which cannot adequately bathe the placental villi in an even slow flow of maternal blood, to meet the even increasing demands of the fetus for oxygen and nutrients. It has been proposed that this reduction in placental perfusion can lead to oxidative stress in the placenta, resulting in the generation of reactive oxygen species [34]. As enhanced interactions between vascular tissue and neutrophils have been demonstrated in ischemic tissues in association with increased generation of reactive oxygen intermediates [35, 36], it is possible that such a mechanism may operate in preeclampsia [14], contributing to the generation to the NETs. In this manner, a cascade, with an endless loop nature, can be envisaged whereby the initial hypoxic condition and associated oxidative stress brought about by inadequate modification of the spiral arteries will lead to neutrophils activation and NETs formation. The presence of these NETs will further hinder blood circulation through the intervillous space, thereby increasing the degree of hypoxia and associated oxidative stress. In addition, it has been proposed that hypoxia promotes release of inflammatory cytokines such IL- 6 and IL-8 by placental trophoblast cells from preeclamptic pregnancies [37]. Consequently, the increase in the STBM release and IL-8 production by the ischemic placenta could attract further additional neutrophils into the placenta, and their subsequent activation would lead to further cycles of recruitment and activation. In this manner, our observations provide a new insight into the generally accepted view of reduced placenta perfusion in preeclampsia.

Our data may, however, also bear insight into a currently less widely accepted new proposal that preeclampsia is not the result of placental hypoxia but involves periods of hypoxia and re-oxygenation, which would be associated with a considerable degree of oxidative stress [34]. This fluctuation in blood flow would be mediated by the rapid generation and clearance of NETs, leading to a sudden influx of oxygen-rich maternal blood into these hypoxic tissues, thereby contributing conditions of oxidative stress. As these alternating conditions of hypoxia/re-oxygenation or oxidative stress are associated with increased apoptosis and aponecrosis of the villous tissue $[6,38]$, this would lead to an increased release of STBM by the ischemic placenta, thereby inducing the generation of more NETs.

The increased presence of these NETs may also explain the increased levels of maternal circulatory cell-free DNA previously observed in preeclampsia $[39,40]$. As these circulatory cell-free DNA levels correlate with disease severity, this would be indicative of increased NETs formation, which would fit well with the proposal that such extracellular entities may play an important role in the underlying etiology of preeclampsia.

\section{Conclusions}

In this review, for the first time, we have described a novel finding of NETs formation in an inflammatory disease like preeclampsia. Considering the present notion of preeclampsia pathogenesis, NETs might have a role in the oxidative stress induction that was observed in its pathogenesis. Further studies are needed to confirm their presence in other inflammatory and autoinflammatory diseases where neutrophil activation has been shown. Understanding the molecular pathways leading to NETs generation after neutrophil activation will give us a better insight into the pathogenesis of inflammatory disorders and might be useful for the therapeutics.

Acknowledgments We would like to thank Mr. Daniel Mathys (ZMB, University of Basel) for his excellent technical assistance with the scanning electron microscopy and Mrs. Vivian Kiefer-Vargas and Mrs. Lisbeth Dudler for their kind help in the preparation of specimens for microscopic analysis. We would also like to thank Dr. Susanne Gatfield for her help with fluorescence microscopy and Dr. Carolyn Troeger for placentae collection.

\section{References}

1. Redman CW, Sargent IL (2005) Latest advances in understanding preeclampsia. Science 308:1592-1594

2. Borzychowski AM, Sargent IL, Redman CW (2006) Inflammation and pre-eclampsia. Semin Fetal Neonatal Med 11:309-316

3. Sacks G, Sargent I, Redman C (1999) An innate view of human pregnancy. Immunol Today 20:114-118

4. Sargent IL, Germain SJ, Sacks GP, Kumar S, Redman CW (2003) Trophoblast deportation and the maternal inflammatory response in pre-eclampsia. J Reprod Immunol 59:153-160

5. Redman CW, Sargent IL (2004) Preeclampsia and the systemic inflammatory response. Semin Nephrol 24:565-570 
6. Huppertz B, Kingdom J, Caniggia I, Desoye G, Black S, Korr H, Kaufmann P (2003) Hypoxia favours necrotic versus apoptotic shedding of placental syncytiotrophoblast into the maternal circulation. Placenta 24:181-190

7. Knight M, Redman CW, Linton EA, Sargent IL (1998) Shedding of syncytiotrophoblast microvilli into the maternal circulation in pre-eclamptic pregnancies. Br J Obstet Gynaecol 105:632-640

8. Smarason AK, Sargent IL, Starkey PM, Redman CW (1993) The effect of placental syncytiotrophoblast microvillous membranes from normal and pre-eclamptic women on the growth of endothelial cells in vitro. Br J Obstet Gynaecol 100:943-949

9. Gupta AK, Rusterholz C, Huppertz B, Malek A, Schneider H, Holzgreve W, Hahn S (2005) A comparative study of the effect of three different syncytiotrophoblast micro-particles preparations on endothelial cells. Placenta 26:59-66

10. Cockell AP, Learmont JG, Smarason AK, Redman CW, Sargent IL, Poston L (1997) Human placental syncytiotrophoblast microvillous membranes impair maternal vascular endothelial function. Br J Obstet Gynaecol 104:235-240

11. Barden A, Graham D, Beilin LJ, Ritchie J, Baker R, Walters BN, Michael CA (1997) Neutrophil CD11B expression and neutrophil activation in pre-eclampsia. Clin Sci (Lond) 92:37-44

12. Clark P, Boswell F, Greer IA (1998) The neutrophil and preeclampsia. Semin Reprod Endocrinol 16:57-64

13. Aly AS, Khandelwal M, Zhao J, Mehmet AH, Sammel MD, Parry S (2004) Neutrophils are stimulated by syncytiotrophoblast microvillous membranes to generate superoxide radicals in women with preeclampsia. Am J Obstet Gynecol 190:252-258

14. Tsukimori K, Maeda H, Ishida K, Nagata H, Koyanagi T, Nakano $\mathrm{H}$ (1993) The superoxide generation of neutrophils in normal and preeclamptic pregnancies. Obstet Gynecol 81:536-540

15. Gryglewski RJ, Palmer RM, Moncada S (1986) Superoxide anion is involved in the breakdown of endothelium-derived vascular relaxing factor. Nature 320:454-456

16. Holzgreve W, Ghezzi F, Di Naro E, Ganshirt D, Maymon E, Hahn S (1998) Disturbed feto-maternal cell traffic in preeclampsia. Obstet Gynecol 91:669-672

17. Lo YM, Lau TK, Zhang J, Leung TN, Chang AM, Hjelm NM, Elmes RS, Bianchi DW (1999) Increased fetal DNA concentrations in the plasma of pregnant women carrying fetuses with trisomy 21. Clin Chem 45:1747-1751

18. Zhong XY, Laivuori H, Livingston JC, Ylikorkala O, Sibai BM, Holzgreve W, Hahn S (2001) Elevation of both maternal and fetal extracellular circulating deoxyribonucleic acid concentrations in the plasma of pregnant women with preeclampsia. Am J Obstet Gynecol 184:414-419

19. Swinkels DW, de Kok JB, Hendriks JC, Wiegerinck E, Zusterzeel PL, Steegers EA (2002) Hemolysis, elevated liver enzymes, and low platelet count (HELLP) syndrome as a complication of preeclampsia in pregnant women increases the amount of cell-free fetal and maternal DNA in maternal plasma and serum. Clin Chem 48:650-653

20. Leung TN, Zhang J, Lau TK, Chan LY, Lo YM (2001) Increased maternal plasma fetal DNA concentrations in women who eventually develop preeclampsia. Clin Chem 47:137-139

21. Zhong XY, Holzgreve W, Hahn S (2002) The levels of circulatory cell free fetal DNA in maternal plasma are elevated prior to the onset of preeclampsia. Hypertens Pregnancy 21:77-83

22. Levine RJ, Qian C, Leshane ES, Yu KF, England LJ, Schisterman EF, Wataganara T, Romero R, Bianchi DW (2004) Two-stage elevation of cell-free fetal DNA in maternal sera before onset of preeclampsia. Am J Obstet Gynecol 190:707-713

23. Hahn S, Holzgreve W (2002) Fetal cells and cell-free fetal DNA in maternal blood: new insights into pre-eclampsia. Hum Reprod Update 8:501-508

24. Brinkmann V, Reichard U, Goosmann C, Fauler B, Uhlemann Y, Weiss DS, Weinrauch Y, Zychlinsky A (2004) Neutrophil extracellular traps kill bacteria. Science 303:1532-1535

25. Wartha F, Beiter K, Normark S, Henriques-Normark B (2007) Neutrophil extracellular traps: casting the NET over pathogenesis. Curr Opin Microbiol 10:52-56

26. Urban CF, Reichard U, Brinkmann V, Zychlinsky A (2006) Neutrophil extracellular traps capture and kill Candida albicans yeast and hyphal forms. Cell Microbiol 8:668-676

27. Buchanan JT, Simpson AJ, Aziz RK, Liu GY, Kristian SA, Kotb M, Feramisco J, Nizet V (2006) DNase expression allows the pathogen group A Streptococcus to escape killing in neutrophil extracellular traps. Curr Biol 16:396-400

28. Beiter K, Wartha F, Albiger B, Normark S, Zychlinsky A, Henriques-Normark B (2006) An endonuclease allows Streptococcus pneumoniae to escape from neutrophil extracellular traps. Curr Biol 16:401-407

29. Lippolis JD, Reinhardt TA, Goff JP, Horst RL (2006) Neutrophil extracellular trap formation by bovine neutrophils is not inhibited by milk. Vet Immunol Immunopathol 113:248-255

30. Lee WL, Grinstein S (2004) Immunology. The tangled webs that neutrophils weave. Science 303:1477-1478

31. Gupta AK, Hasler P, Holzgreve W, Gebhardt S, Hahn S (2005) Induction of neutrophil extracellular DNA lattices by placental microparticles and IL-8 and their presence in preeclampsia. Hum Immunol 66:1146-1154

32. Huppertz B, Kingdom JC (2004) Apoptosis in the trophoblastrole of apoptosis in placental morphogenesis. J Soc Gynecol Investig 11:353-362

33. Lim KH, Zhou Y, Janatpour M, McMaster M, Bass K, Chun SH, Fisher SJ (1997) Human cytotrophoblast differentiation/invasion is abnormal in pre-eclampsia. Am J Pathol 151:1809-1818

34. Roberts JM, Hubel CA (2004) Oxidative stress in preeclampsia. Am J Obstet Gynecol 190:1177-1178

35. Canas PE (1999) The role of xanthine oxidase and the effects of antioxidants in ischemia reperfusion cell injury. Acta Physiol Pharmacol Ther Latinoam 49:13-20

36. Zweier JL, Talukder MA (2006) The role of oxidants and free radicals in reperfusion injury. Cardiovasc Res 70:181-190

37. Bowen RS, Gu Y, Zhang Y, Lewis DF, Wang Y (2005) Hypoxia promotes interleukin- 6 and -8 but reduces interleukin-10 production by placental trophoblast cells from preeclamptic pregnancies. J Soc Gynecol Investig 12:428-432

38. Ishihara $\mathrm{N}$, Matsuo $\mathrm{H}$, Murakoshi $\mathrm{H}$, Laoag-Fernandez JB, Samoto T, Maruo T (2002) Increased apoptosis in the syncytiotrophoblast in human term placentas complicated by either preeclampsia or intrauterine growth retardation. Am J Obstet Gynecol 186:158-166

39. Lo YM, Leung TN, Tein MS, Sargent IL, Zhang J, Lau TK, Haines CJ, Redman CW (1999) Quantitative abnormalities of fetal DNA in maternal serum in preeclampsia. Clin Chem 45:184-188

40. Zhong XY, Holzgreve W, Hahn S (2001) Circulatory fetal and maternal DNA in pregnancies at risk and those affected by preeclampsia. Ann N Y Acad Sci 945:138-140 